\title{
MEFV Variants in Patients with PFAPA Syndrome in Japan
}

\author{
Shoichiro Taniuchi ${ }^{*}, 1$, Ryuta Nishikomori ${ }^{2}$, Anna Iharada ${ }^{1}$, Shoji Tuji ${ }^{1}$, Toshio Heike ${ }^{2}$ and \\ Kazunari Kaneko ${ }^{1}$
}

\author{
${ }^{I}$ Department of Pediatrics, Kansai Medical University, Japan \\ ${ }^{2}$ Department of Pediatrics, Kyoto University, Japan
}

\begin{abstract}
Background: The pathogenesis of PFAPA (periodic fever, aphthous stomatitis, pharyngitis, adenitis) syndrome is unknown as yet. In order to understand whether genes implicated in other auto-inflammatory diseases might be involved in the pathogenesis of PFAPA, all variants in the genes causing familial Mediterranean fever (FMF), tumor necrosis factor (TNF) receptor-associated periodic syndrome (TRAPS), and Hyper IgD syndrome were analyzed in children with PFAPA.

Patients and Methods: All variants in MEFV, TNFRSF1A, and MVK were analyzed in 20 patients with PFAPA. PFAPA were diagnosed by previous published criteria. The findings of all analyses in PFAPA patients were compared with those of unaffected normal subjects $(\mathrm{n}=62)$.

Results: In the 13 children of 20 with PFAPA, the heterozygous variants of $M E F V$ (5 patients: E148Q-L110P, 2 patients: E148Q, 1 patient: $E 148 Q-L 110 P / E 148 Q$, 1 patient: E148Q-P369S-R408Q-E84K, 1 patient: E148Q-L110P-P369S-A408G, 1 patient: $R 202 Q$, 1 patient: $P 115 R$ ) were found. No variants belonging to TNFRSF1A or MVK were detected in children with PFAPA. The frequency of the E148Q-L110P variants in children with PFAPA was significantly higher than that observed in unaffected normal subjects (7/20 versus 8/62). The duration of the episodes of illness in PFAPA children with $M E F V$ variants was shorter than that of patients without variants.
\end{abstract}

Conclusion: Genes involved in the development and progression of $M E F V$ may affect the incidence and the phenotype of PFAPA in children.

Keywords: PFAPA, MEFV, FMF, Variant, Japanese.

\section{INTRODUCTION}

In 1987 [1], Marshall firstly described, the PFAPA (periodic fever, aphthous stomatitis, pharyngitis and adenitis) syndrome, which is characterized by recurrent episodes of fever associated with cervical adenitis, pharyngitis and aphthous stomatitis. The prognosis of this disease has been reported to be better than that of another autoinflammatory diseases [2]. Corticosteroids are effective in controlling episodes of illness in PFAPA, but they do not cure the ailments or prevent recurrence of the symptoms of this syndrome [3]. Interventions like tonsillectomy and administration of $\mathrm{H}_{2}$ blockers have been reported to be partially effective for prophylaxis [3]. However the complete pathogenesis of PFAPA is unknown yet, and hence the therapeutic regimens have not yet been established for PFAPA [4].

Familial Mediterranean fever (FMF) is a recessively inherited disorder characterized by acute attacks of fever, and serositis usually lasting for $1-3$ days. FMF is caused by

*Address correspondence to this author at the Department of Pediatrics, Kansai Medical University, Fumizonocho 10-15, Moriguchi, Osaka 5708506, Japan; Tel: (+81)-6-6992-1001; Fax: (+81)-6-6992-9355;

E-mail: taniuchi@takii.kmu.ac.jp mutations in the ME diterranean FeVer gene $(M E F V)$, which encodes the protein pyrin (marenostrin)1[5,6]. Colchicine has been shown to be effective for prophylaxis in only $90 \%$ of the patients with FMF $[7,8]$.

Recent studies have described that heterozygous variants of the $M E F V$ gene were found in patients with PFAPA [911]. However, it still remains unclear whether these variants are the causative factors of PFAPA.

The purpose of this study was to understand whether heterozygous variants of $M E F V$ may be associated with the onset of PFAPA. We have also tried to understand whether these mutations act as accessory factors and modify the phenotype of patients with PFAPA.

\section{PATIENTS AND METHODS}

Twenty children with frequent PFAPA episodes who visited our pediatric outpatient clinic were consecutively selected over a 5-year period (from January 2005 to January 2010). The diagnosis of PFAPA syndrome was established using previously established criteria $[1,3,9]$. These criteria include recurring fevers associated with exudative tonsillitis, negative throat culture, and possibly, aphthous stomatitis and cervical lymphadenopathy. The additional clinical criteria included completely asymptomatic intervals between the episodes, normal growth and development and exclusion of 
FMF, Behcet' $s$ disease, and cyclic neutropenia. Oral low dose of prednisolone $(0.3-0.5 \mathrm{mg} / \mathrm{kg} / \mathrm{dose}, 1$ or 2 doses per day) was effective on all enrolled patients. $M E F V$, mevalonate kinase $(M V K)$, and tumor necrosis factor receptor superfamily, member $1 \mathrm{~A}$ (TNFRSF1A) genes of all enrolled patients were sequenced. After obtaining a written informed consent approved by the Institutional Review Board of Kyoto University, peripheral blood was collected from all patients, and, if needed, their family members. Genomic DNA was extracted, and all the exons including exon-intron junctions of the $M V K, M E F V$, and TNFRSF1A genes were amplified by polymerase chain reaction and then sequenced using the ABI3130.

The results are shown as a mean $\pm \mathrm{SD}$ or proportion, as appropriate. Differences between the groups in discrete variables were evaluated using Fisher's exact test at 5\% significance.

Two-sided $P$ values were adjusted for multiplicity using Hochberg's method.

Comparisons of continuous variables were done using unpaired Student's $t$-test. All $P$ values given are 2 -sided. $P$ values less than 0.05 were considered significant. Statistical calculation was conducted by SAS version 9.1.3.

\section{RESULTS}

Twenty patients (9 boys, 11 girls) diagnosed with PFAPA were followed up in our clinic. Thirteen of these patients had a single $M E F V$ ( $\mathrm{M}^{+}$group). No variant of TNFRSF1A and MVK was detected in all patients. The genotypes of the $M E F V$ gene in the 13 patients are seen in Table 1. The most common $M E F V$ variant patterns seen were $E 148 Q-L 110 P$ (5 patients) and $E 148 Q$ (2 patients). One patient was homozygous of $E 148 Q$ and heterozygous of $L 110 P$ of $M E F V$. One patient was heterozygous of E148Q$P 369 S-R 408 Q$. One patient was heterozygous for E148QP369S-R408Q-E84K. One patient had E148Q-L110P$P 369 S-R 408 Q$. The minor variants, $P 115 R$ and $R 202 Q$, were detected in 2 patients. More than $2 M E F V$ variants were on 1 allele in all PFAPA patients. In 7 patients, no $M E F V$ mutations were found. The allele frequencies of $E 148 Q$, $L 110 P, P 369 S, R 408 Q$ and G304R in 20 PFAPA patients were $0.3,0.175,0.075,0.075$ and 0 , respectively (Table 3 ).

Table 1. The Genotypes of MEFV Genes of 13 Patients with PFAPA

\begin{tabular}{|c|c|}
\hline MEFV Variant & No. of Patients \\
\hline \hline$E 148 Q-L 110 P$ & 5 \\
\hline$E 148 Q$ & 2 \\
\hline$E 148 Q-L 110 P / E 148$ & 1 \\
\hline$E 148 Q-P 369 S-R 408 Q$ & 1 \\
\hline$E 148 Q-P 369 S-R 408 Q-E 84 K$ & 1 \\
\hline$E 148 Q-L 110 P-P 369 S-R 408 Q$ & 1 \\
\hline$R 202 Q$ & 1 \\
\hline$P 115 R$ & 1 \\
\hline
\end{tabular}

Clinical and laboratory data were compared between $M E F V$ positive group $(\mathrm{n}=13)$ and negative group $(\mathrm{n}=7)$ and are presented in Table 2. Patients carrying an $M E F V$ variant showed shorter duration of episodes of illness than patients without variants $(3.6 \pm 0.86$ days versus $5.3 \pm 1.89$ days,

Table 2. Clinical Characteristics of PFAPA Patients with Variants in the MEFV Gene Compared with those of PFAPA Patients without $M E F V$ Variants

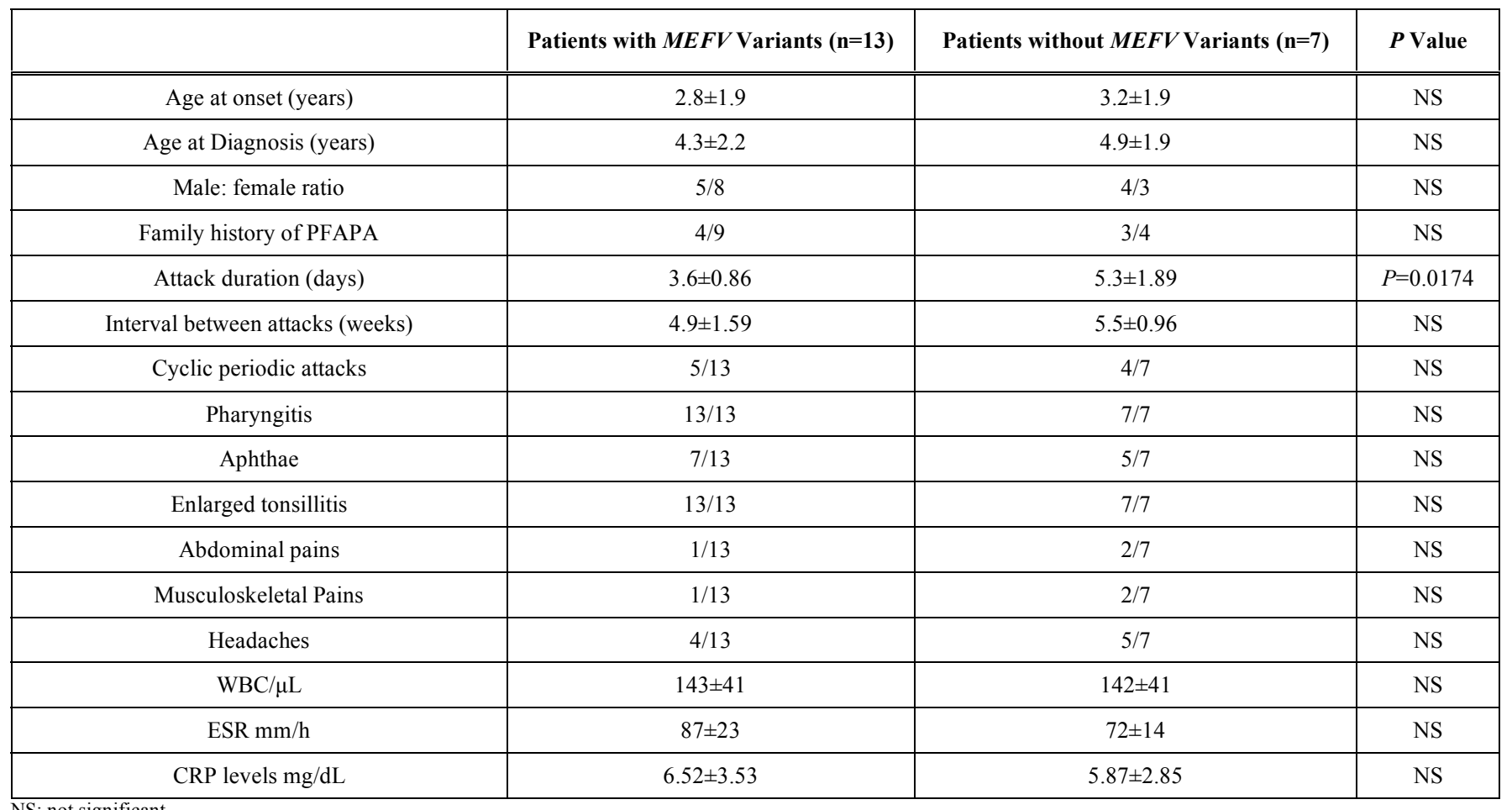


$p=0.0174)$. No significant differences in all other clinical and laboratory data were found between the 2 groups (Table 2 ).

We also analyzed all sequences of $M E F V$ genes in normal Japanese subjects $(n=62)$. These individuals were healthy adult volunteers and had no recurrent episodes of fever. There was no difference in recruitment between the PFAPA patients and the control group. A comparison of these results between normal and PFAPA subjects is shown in Table 3. In normal individuals, no significant allele frequencies were observed for the 4 variants found in PFAPA patients. In addition, the frequencies of E148Q$L 110 P$ and $P 369 S-R 408 Q$ in the 2 groups were compared. A significant difference in the frequency of these variants was observed between the 2 groups (Table 4, $p=0.043$ and $p=$ 0.026 , respectively).

Table 3. Allele Frequencies of $M E F V$ Variants in PFAPA Subjects and Normal Unaffected Subjects

\begin{tabular}{|c|c|c|c|}
\hline Variant & $\begin{array}{c}\text { PFAPA Subjects } \\
(\mathbf{n}=\mathbf{4 0 )}\end{array}$ & $\begin{array}{c}\text { Unaffected Subjects } \\
(\mathbf{n}=\mathbf{1 2 4})\end{array}$ & $\boldsymbol{P}$ Value \\
\hline \hline$E 148 Q$ & $30.0 \%$ & $18.5 \%$ & NS \\
\hline$L 110 P$ & $17.5 \%$ & $6.5 \%$ & NS \\
\hline$G 304 R$ & $0.0 \%$ & $3.2 \%$ & NS \\
\hline
\end{tabular}

Table 4. Frequencies of $M E F V$ Variants in PFAPA Subjects and Normal Unaffected Subjects

\begin{tabular}{|c|c|c|c|}
\hline Variant & $\begin{array}{c}\text { PFAPA Subjects } \\
(\mathbf{n}=\mathbf{2 0})\end{array}$ & $\begin{array}{c}\text { Unaffected Subjects } \\
(\mathbf{n}=\mathbf{6 2})\end{array}$ & $\boldsymbol{P}$ Value \\
\hline \hline$E 148 Q-L 110 P$ & $35 \%$ & $13 \%$ & $P=0.043$ \\
\hline
\end{tabular}

\section{DISCUSSION}

We studied 20 patients with PFAPA who were diagnosed by Marshall criteria [1]. Our aim was to access the roles of the predominant variants in genes that cause other febrile illnesses like FMF, TNF receptor-associated periodic syndrome (TRAPS) and the MVK deficiency. We did not find any incidence of variants of TRAPS and MVK deficiency in PFAPA patients. However several heterogeneous variants of $M E F V$ were detected in 13 out of 20 patients with PFAPA. We analyzed the frequency of the E148Q-L110P and P369S$R 408 Q$ variants in PFAPA and control subjects. Our analyses indicate that the incidence of these 2 variants is significantly higher in patients with PFAPA than in normal individuals.

Amongst autoinflammatory disease, only PFAPA syndrome has been described as a non-inherited syndrome, since familial inheritance has not been reported in previous studies $[3,12,13]$. However some studies have reported familial cases that included siblings or a sibling and the sibling's mother [14-16]. Therefore, the hereditary nature of this syndrome is still a matter of debate. With respect to the genetic factors that may cause the PFAPA syndrome, one study has strongly argued against the involvement of the $M E F V$ gene [10], but another article [11] has shown that mutations of the $M E F V$ genes were found in $27 \%$ of cases diagnosed with PFAPA syndrome on the basis of Marshall's clinical criteria. Our observations of the high frequency (65\%) of $M E F V$ variants are in agreement with that reported by Dagan [11]. The differences in the findings may be attributed to the ethnic differences between the individuals studied, the small sized of the study, and the study population that was selected.

The $L 110 P$ variant, which is located in exon 2, was first reported in FMF patients in 2000 [17], and to date, several compound heterozygotes with other variants have been reported even in Japan $[18,19]$. In contrast, although the role of the $E 148 Q$ variant, which is located in exon 2, in FMF patients was controversial, a recent study concluded that the variant is just a benign polymorphism [20]. In a Japanese study [18] of FMF patients, the most frequently observed $M E F V$ variants observed were E148Q/M694I (25.0\%), M694I (17.5\%), and L110P/E148Q/M694I (17.5\%). However, no patients had the $M 694 \mathrm{~V}$ variant. These patterns are quite different from those in Mediterranean patients with FMF. The study also reported that the allele frequencies of $E 148 Q$, M694I, and $L 110 P$ were $0.44,0.35$, and 0.31 , respectively, and that these frequencies were significant difference from those seen in healthy controls. In our study, the allele frequencies of $E 148 Q, L 110 P$, and $G 304$ were $0.3,0.175$, and 0 , respectively, and these frequencies did not differ significantly between patients and healthy controls. No mutation at the M694I was detected in our cohorts of patients and controls. The allele frequency of $L 110 P$ is higher in patients with PFAPA than in healthy controls; however, the difference is not significant. The frequency of the E148Q$L 110 P$ variant combination is significantly higher in the PFAPA group than in the healthy control group. If the $E 148 Q$ variant is non-functional, the L110P variant may be associated with the onset of PFAPA syndrome.

In several types of inflammatory such as Behcet's disease [21], Crohn's disease [22], ulcerative colitis [23], HenochSchönlein purpura $[24,25]$ and the co-incidence of FMF variants has been investigated. These studies show increased incidence of genes involved in FMF in patients with these autoinflammatory diseases as well as the increased severity of the symptoms of each disease. On the other, in the patients with asthma, the incidence of FM mutation was decreased and the lower incidence correlated with reduced severity of symptoms [26]. Thus, FMF variants may affect the transition from Th2 to Th1 polarity in each disease. According to Berkun's study [27], PFAPA episodes in carriers of $M E F V$ variants were shorter compared to those in patients without variants. In $M E F V$ variant-positive patients, the regular cyclic pattern of attacks and the occurrence of oral aphthae was lower than those in patients without $M E F V$ variants. In the present study, we found that the only affected variable was the duration of PFAPA episodes. Although no significant differences were observed in the regular cyclic pattern of attacks and the occurrence of oral aphthae between the 2 groups, the duration of PFAPA episodes was shorter in the variant-positive group than in the variant-negative group.

Taken together, these results show that the $M E F V$ gene may not affect the onset of several autoinflammatory diseases, but is likely to modify the intensity and the displayed phenotype in terms of disease symptoms. 
In conclusion, the $M E F V$ variants, viz. E148Q-L110P, $P 369-R 408 Q$ may be associated with the onset of PFAPA, and some $M E F V$ variants may affect the phenotype of PFAPA.

\section{CONFLICT OF INTEREST}

The authors confirm that this article content has no conflict of interest.

\section{ACKNOWLEDGEMENTS}

The study was supported by the Mami Mizutani Foundation.

\section{REFERENCES}

[1] Marshall GS, Edwards KM, Lawton AR. PFAPA syndrome. Pediatr Infect Dis J 1989; 8: 658-9.

[2] Wurster VM, Carlucci JG, Feder HM Jr, Edwards KM. Long-term follow-up of children with periodic fever, aphthous stomatitis, pharyngitis, and cervical adenitis syndrome. J Pediatr 2011; 159: 95864.

[3] Thomas KT, Feder HM Jr, Lawton AR, Edwards KM. Periodic fever syndrome in children. J Pediatr 1999; 135: 15-21.

[4] Feder HM, Salazar JC. A clinical review of 105 patients with PFAPA (a periodic fever syndrome). Acta Paediatr 2010; 99: 178-84.

[5] The International FMF Consortium. Ancient missense mutations in a new member of the RoRet gene family are likely to cause familial Mediterranean fever. Cell 1997; 90: 797-807.

[6] French FMF Consortium. A candidate gene for familial Mediterranean fever. Nat Genet 1997; 17: 25-31.

[7] Medlej-Hashim M, Loiselet J, Lefranc G, Mégarbané A . [Familial Mediterranean Fever (FMF): from diagnosis to treatment. Sante 2004; 14: 261-6.

[8] Soylemezoglu O, Arga M, Fidan K, et al. Unresponsiveness to colchicine therapy in patients with familial Mediterranean fever homozygous for the M694V mutation. J Rheumatol 2010; 37: 182-9.

[9] Padeh S, Brezniak N, Zemer D, et al. Periodic fever, aphthous stomatitis, pharyngitis, and adenopathy syndrome: clinical characteristics and outcome. J Pediatr 1999; 135: 98-101.

[10] Cazeneuve C, Geneviève D, Amselem S, Hentgen V, Hau I, Reinert P. MEFV gene analysis in PFAPA. J Pediatr 2003; 143: 140-1.

[11] Dagan E, Gershoni-Baruch R, Khatib I, Mori A, Brik R. MEFV, TNF1rA, CARD15 and NLRP3 mutation analysis in PFAPA. Rheumatol Int 2010; 30: 633-6.

[12] Feder HM Jr, Bialecki CA. Periodic fever associated with aphthous stomatitis, pharyngitis and cervical adenitis. Pediatr Infect Dis J 1989; 8: $186-7$.
[13] Tasher D, Somekh E, Dalal I. PFAPA syndrome: new clinical aspects disclosed. Arch Dis Child 2006; 91: 981-4.

[14] Sampaio IC, Rodrigo MJ, Monteiro Marques JG. Two siblings with periodic fever, aphthous stomatitis, pharyngitis, adenitis (PFAPA) syndrome. Pediatr Infect Dis J 2009; 28: 254-5.

[15] Valenzuela PM, Majerson D, Tapia JL, Talesnik E. Syndrome of periodic fever, aphthous stomatitis, pharyngitis, and adenitis (PFAPA) in siblings. Clin Rheumatol 2009 ; 28: 1235-7.

[16] Adachi M, Watanabe A, Nishiyama A, et al. Familial cases of periodic fever with aphthous stomatitis, pharyngitis, and cervical adenitis syndrome. J Pediatr 2011; 158: 155-9.

[17] Domingo C, Touitou I, Bayou A, et al. Familial Mediterranean fever in the 'Chuetas' of Mallorca: a question of Jewish origin or genetic heterogeneity. Eur J Hum Genet 2000; 8: 242-6.

[18] Tsuchiya-Suzuki A, Yazaki M, Nakamura A, et al. Clinical and genetic features of familial Mediterranean fever in Japan. J Rheumatol 2009; 36: 1671-6

[19] Tomiyama $\mathrm{N}$, Higashiuesato $\mathrm{Y}$, Oda $\mathrm{T}$, et al. $M E F V$ mutation analysis of familial Mediterranean fever in Japan. Clin Exp Rheumatol 2008; 26: 13-7.

[20] Tchernitchko D, Legendre M, Cazeneuve C, Delahaye A, Niel F, Amselem S. The E148Q MEFV allele is not implicated in the development of familial Mediterranean fever. Hum Mutat 2003; 22: 339-40.

[21] Rabinovich E, Shinar Y, Leiba M, Ehrenfeld M, Langevitz P, Livneh A. Common FMF alleles may predispose to development of Behcet's disease with increased risk for venous thrombosis. Scand J Rheumatol 2007; 36: 48-52.

[22] Uslu N, Yüce A, Demir H, et al. The association of inflammatory bowel disease and Mediterranean fever gene (MEFV) mutations in Turkish children. Dig Dis Sci. 2010; 55: 3488-94.

[23] Giaglis S, Mimidis K, Papadopoulos V, et al. Increased frequency of mutations in the gene responsible for familial Mediterranean fever $(M E F V)$ in a cohort of patients with ulcerative colitis: evidence for a potential disease-modifying effect? Dig Dis Sci 2006; 51: 687-92.

[24] Gershoni-Baruch R, Broza Y, Brik R. Prevalence and significance of mutations in the familial Mediterranean fever gene in HenochSchönlein purpura. J Pediatr 2003; 143: 658-61.

[25] Ozçakar ZB, Yalçinkaya F, Cakar N, et al. MEFV mutations modify the clinical presentation of Henoch-Schönlein purpura. J Rheumatol 2008; 35: 2427-9.

[26] Rabinovitch E, Harats D, Yaron P, et al. Familial Mediterranean fever gene and protection against asthma. Ann Allergy Asthma Immunol 2007; 99: 517-21

[27] Berkun Y, Levy R, Hurwitz A, et al. The familial Mediterranean fever gene as a modifier of periodic fever, aphthous stomatitis, pharyngitis, and adenopathy syndrome. Semin Arthritis Rheum 2011; 40: 467-72. 